Hong-Ru Dong* and Jian-Guo Wu

\title{
Design, synthesis and anticancer activity evaluation of aziridine-1,2,3-triazole hybrid derivatives
}

https://doi.org/10.1515/hc-2017-0144

Received August 1, 2017; accepted December 19, 2017; previously published online March 30, 2018

\begin{abstract}
New 1-aryl-4-[(aziridine-1-yl)diarylmethyl]-5-methyl-1H-1,2,3-triazole derivatives $7 \mathbf{a}-\mathbf{i}$ were synthesized by a one-pot reaction of diaryl-(1-aryl-5-methyl-1H-1,2,3triazol-4-yl)methanols $\mathbf{6 a - i}$ derived from 1-aryl-5-methyl-1H-1,2,3-triazole-4-carboxylic acids 4a-c. Structures of compounds $7 \mathbf{a}-\mathbf{i}$ were confirmed by analysis of proton nuclear magnetic resonance ( $\left.{ }^{1} \mathrm{H} \mathrm{NMR}\right)$ and carbon-13 nuclear magnetic resonance $\left({ }^{13} \mathrm{C} \mathrm{NMR}\right)$, mass spectrometry (MS) and infrared (IR) data. The structure of compound 7f was studied by X-ray diffraction analysis. The anticancer activities of compounds $7 \mathbf{a}-\mathbf{i}$ against human leukemia HL-60 cells and human hepatoma G2 cells were evaluated. Some of the compounds are highly active.
\end{abstract}

Keywords: anticancer; aziridine; human hepatoma G2; human leukemia HL-60; $1 H$-1,2,3-triazole.

\section{Introduction}

A vast majority of biologically important molecules are heterocyclic compounds [1-3]. The biological properties of aziridine-containing compounds such as azicemicins [4], miraziridine [5, 6], azinomycins [7], ficellomycin [8], FR-900482 [9] and mitomycins [10] are of significant interest (Figure 1). In particular, mitomycin $\mathrm{C}$ has a broad activity against a range of tumors and has been applied clinically since the 1960s. 1,2,3-Triazoles are also pharmacologically important molecules that show anticancer [11-13], antibacterial [14, 15], antifungal [16], antiviral [17], anti-inflammatory and analgesic activities [18] (Figure 2). However, there is little information describing compounds containing aziridine and 1,2,3-triazole moieties in one molecule. Such compounds are the subject of this report.

*Corresponding author: Hong-Ru Dong, School of Chemical Engineering, Lanzhou University of Arts and Science, Lanzhou 730000, Gansu, P.R. China, e-mail: donghr@luas.edu.cn Jian-Guo Wu: School of Chemical Engineering, Lanzhou University of Arts and Science, Lanzhou 730000, Gansu, P.R. China

\section{Results and discussion}

1-Aryl-4-[(aziridin-1-yl)diaryl-methyl]-5-methyl-1H-1,2,3-triazoles 7a-i were synthesized from diaryl-1H-1,2,3-triazol-4-yl) methanols $\mathbf{6 a - i}$. The synthetic route is shown in Scheme 1. Details of the synthesis of compounds $\mathbf{6 a - i}$ starting with anilines $\mathbf{1}$ through intermediaries $\mathbf{4}$ and $\mathbf{5}$ were described in our previous report [19]. Structures of products $7 \mathbf{a}-\mathbf{i}$ were deduced by spectral methods. In particular, a strong absorption at $3320-3520 \mathrm{~cm}^{-1}$ for a hydroxy group, observed in the infrared (IR) spectra of substrates $\mathbf{6 a - i}$, is absent in the IR spectra of products $7 \mathbf{a}-\mathbf{i}$. The proton nuclear magnetic resonance ( ${ }^{1} \mathrm{H}$ NMR) spectra of compounds $7 \mathbf{a}-\mathbf{i}$ show signals of the aziridine protons at $\delta 1.80-1.88$ and $\delta 1.15-1.33$, as expected. The structure of compound $\mathbf{7 f}$ was additionally determined by X-ray diffraction analysis (Figure 3). Compound 7f (CCDC deposit No 775599), $\mathrm{C}_{25} \mathrm{H}_{24} \mathrm{~N}_{4}$, MW 380.48, crystallizes in the triclinic space group $P \overline{1}$ with unit cell parameters $\mathrm{a}=9.3100(7), \mathrm{b}=13.8263(11), \mathrm{c}=17.4605(15) \AA$, $\alpha=107.402(4), \beta=92.607(4), \gamma=92.829(4)^{\circ}, \mathrm{V}=2137.8(3) \AA^{3}$, $\mathrm{Z}=4, \mathrm{Dx}=1.182 \mathrm{mg} / \mathrm{cm}^{3}$. The final $\mathrm{R}$ value was 0.0558 .

\section{Conclusions}

New 1-aryl-4-[(aziridin-1-yl)diaryl-methyl]-5-methyl-1H-1,2,3triazoles $\mathbf{7 a - i}$ were synthesized and their anticancer activities were evaluated. The anticancer activity was investigated under our previously reported conditions $[20,21]$ using the MTT assay. Compounds $\mathbf{7 b}, \mathbf{c}$ and $\mathbf{7 i}$ are highly active against human leukemia cells and compounds 7c,d, 7f,g inhibit human hepatoma G2 cells.

\section{Experimental}

${ }^{1} \mathrm{H}$ NMR spectra were obtained in deuterated chloroform $\left(\mathrm{CDCl}_{3}\right)$ at $300 \mathrm{MHz}$. Starting materials 4-6 were synthesized as previously reported [19].

\section{Synthesis of compounds $7 a-i$}

Dry hydrogen chloride was bubbled through a hot solution of compounds $6 \mathbf{a}-\mathbf{i}(0.04 \mathrm{~mol})$ in benzene $(20 \mathrm{~mL})$ in a $50-\mathrm{mL}$ flask equipped 
<smiles></smiles>

Azicemicin $\mathrm{A}: \mathrm{R}=\mathrm{H}$ Azicemicin $\mathrm{B}: \mathrm{R}=\mathrm{Me}$<smiles>COc1cc(C(=O)O[C@H](C(=O)NC(C(=O)NCC(C)=O)=C2[C@H](OC(C)=O)[C@@H](O)C3CN23)[C@]2(C)CO2)c2cccc(C)c2c1</smiles>

Azinomycin A<smiles>CC[C@H](NC(=O)C[C@@H](O)[C@H](CC(C)C)NC(=O)[C@H](CC(C)C)NC(=O)C1N[C@H]1C(=O)O)C(=O)N[C@@H](/C=C/C(=O)O)CCCNC(=N)N</smiles>

Miraziridine<smiles>CC(C)C(N)C(=O)NC(C(=O)O)[C@@H]1CC(NC(=N)N)C=[V]1</smiles>

Ficellomycin<smiles></smiles><smiles>[X]C1=C(C)C(=O)C2=C(C1=O)C(COC(N)=O)C1(OC)C3C(CN23)N1C</smiles>

FR-900482 R $=\mathrm{CHO}$

Mitomycin $\mathrm{A}: \mathrm{X}=\mathrm{OMe} ; \mathrm{R}=\mathrm{H}$ FR-66979 R $=\mathrm{CH}_{2} \mathrm{OH}$ Mitomycin $\mathrm{C}: \mathrm{X}=\mathrm{NH}_{2} ; \mathrm{R}=\mathrm{H}$

Figure 1 Fused aziridine-containing natural products.

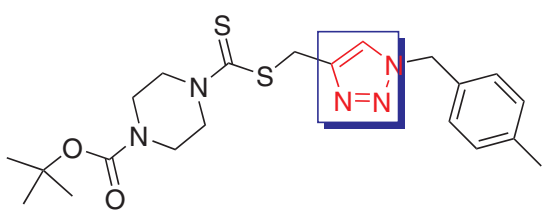<smiles>CCC(O)(Cn1nccc1CCCCn1ccc(=O)[nH]c1=O)c1ccc(F)c(OCC2CC2)c1</smiles>

Figure 2 Anticancer compounds containing 1,2,3-triazole.

with an automatic water separator, a reflux condenser and a calcium chloride guard-tube. After $1 \mathrm{~h}$, the solution was concentrated to $10 \mathrm{~mL}$ and treated with aziridine $(0.06 \mathrm{~mol})$ with rapid stirring followed by the addition of triethylamine $(0.08 \mathrm{~mol})$. After the mixture was stirred at $45^{\circ} \mathrm{C}$ for an additional $1 \mathrm{~h}$, the solvent was evaporated and the residue was crystallized from ethyl acetate or purified by column chromatography on silica gel and eluted with ethyl acetate/ petroleum ether.

4-[(Aziridin-1-yl)-bis(4-chlorophenyl)methyl]-5-methyl-1-(4chlorophenyl)-1H-1,2,3-triazole (7a) White flakes; yield 86\%; mp 90-92 ${ }^{\circ} \mathrm{C}$; ${ }^{1} \mathrm{H}$ NMR: $\delta 7.52$ (d, $\left.1 \mathrm{H}, J=3 \mathrm{~Hz}\right), 7.50$ (d, $\left.1 \mathrm{H}, J=3 \mathrm{~Hz}\right), 7.48$ (d, $2 \mathrm{H}, J=3 \mathrm{~Hz}$ ), 7.45 (d, 2H, $J=3 \mathrm{~Hz}$ ), 7.40 (d, 1H, $J=3.6 \mathrm{~Hz}$ ), 7.37 (d, $1 \mathrm{H}, J=3 \mathrm{~Hz}$ ), 7.29 (d, $2 \mathrm{H}, J=3 \mathrm{~Hz}$ ), 7.26 (d, $2 \mathrm{H}, J=3.6 \mathrm{~Hz}$ ), 2.10 (s, 3H), 1.81 (s, $2 \mathrm{H}), 1.15$ (s, 2H); ${ }^{13} \mathrm{C}$ NMR ( $\left.\mathrm{CDCl}_{3}-\mathrm{d} 1\right), \delta$ 145.7, 141.6, 135.6, 134.7, 133.1, 132.9, 130.4, 129.6, 127.8, 126.8, 69.3, 21.7, 10.6; MS: $\mathrm{m} / z 468\left(\mathrm{M}^{+}\right.$,
0.39), 428 (6), 426 (6), 154 (32), 152 (100); IR: 3425, 3061, 2987, 2926, 2844, 1734, 1496, 1442, 1422, 1400, 1264, 1117, 1092, 1010, 922, 867, 829, $818 \mathrm{~cm}^{-1}$. HRMS (ESI). Calcd for $\mathrm{C}_{24} \mathrm{H}_{19} \mathrm{Cl}_{3} \mathrm{~N}_{4},[\mathrm{M}+\mathrm{H}]^{+}: \mathrm{m} / z 469.0748$. Found: $m / z 469.0755$.

4-[(Aziridin-1-yl)-bis(3-chlorophenyl)methyl]-5-methyl-1-(4chlorophenyl)-1H-1,2,3-triazole (7b) White flakes; yield 65\%; mp 96-98 ${ }^{\circ} \mathrm{C}$; ${ }^{1} \mathrm{H}$ NMR: $\delta 7.61$ (s, 2H), 7.52 (q, 2H, $J=2.4 \mathrm{~Hz}$ ), $7.38(\mathrm{~m}, 4 \mathrm{H})$, 7.23 (m, 4H), 2.04 (s, 3H), 1.84 (d, $2 \mathrm{H}, J=2 \mathrm{~Hz}), 1.17$ (d, $2 \mathrm{H}, J=2 \mathrm{~Hz}) ;{ }^{13} \mathrm{C}$ NMR (CDCl $-\mathrm{d} 1), \delta 145.3,144.8,135.6,134.7,133.7,133.2,129.6,128.9$, 128.8, 127.4, 127.3, 126.8, 69.5, 21.8, 10.6; MS: $m / z 468\left(\mathrm{M}^{+}, 0.71\right), 428$ (5), 154 (27), 152 (100), 111 (29), 75 (12); IR: 3449, 3088, 3063, 2990, 2931, 2869, 1734, 1590, 1572, 1496, 1469, 1422, 1368, 1256, 1202, 1148, 1120, 1091, 1049, 1006, 978, 940, 893, 875, 845, 785, 753, 728, 699, $681 \mathrm{~cm}^{-1}$; HRMS (ESI). Calcd for $\mathrm{C}_{24} \mathrm{H}_{19} \mathrm{Cl}_{3} \mathrm{~N}_{4},[\mathrm{M}+\mathrm{H}]^{+}: \mathrm{m} / z 469.0748$. Found: $m / z$ 469.0751. 


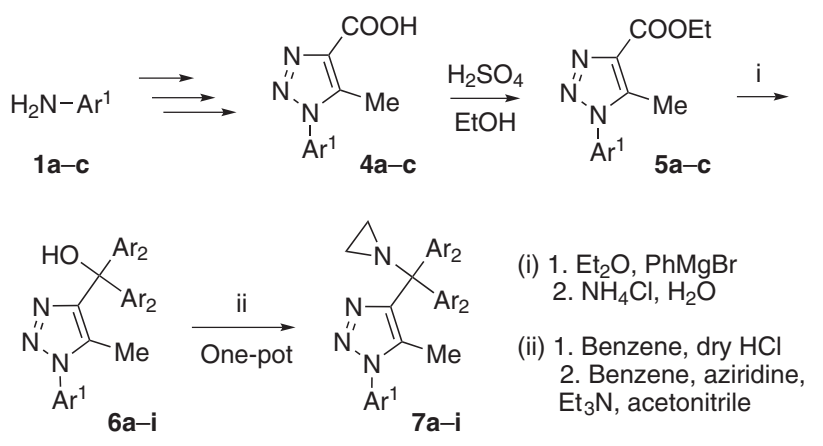

7a: $\mathrm{Ar}^{1}=4-\mathrm{ClC}_{6} \mathrm{H}_{4} ; \mathrm{Ar}^{2}=4-\mathrm{ClC}_{6} \mathrm{H}_{4}$ 7b: $\mathrm{Ar}^{1}=4-\mathrm{ClC}_{6} \mathrm{H}_{4} ; \mathrm{Ar}^{2}=3-\mathrm{ClC}_{6} \mathrm{H}_{4}$ 7c: $\mathrm{Ar}^{1}=4-\mathrm{ClC}_{6} \mathrm{H}_{4} ; \mathrm{Ar}^{2}=\mathrm{Ph}$

$7 \mathrm{~d}: \mathrm{Ar}^{1}=4-\mathrm{MeC}_{6} \mathrm{H}_{4} ; \mathrm{Ar}^{2}=4-\mathrm{ClC}_{6} \mathrm{H}_{4}$ 7e: $\mathrm{Ar}^{1}=4-\mathrm{MeC}_{6} \mathrm{H}_{4} ; \mathrm{Ar}^{2}=3-\mathrm{ClC}_{6} \mathrm{H}_{4}$

7f: $\mathrm{Ar}^{1}=4-\mathrm{MeC}_{6} \mathrm{H}_{4} ; \mathrm{Ar}^{2}=\mathrm{Ph}$ 7g: $\mathrm{Ar}^{1}=\mathrm{Ph} ; \mathrm{Ar}^{2}=4-\mathrm{ClC}_{6} \mathrm{H}_{4}$ 7h: $\mathrm{Ar}^{1}=\mathrm{Ph} ; \mathrm{Ar}^{2}=3-\mathrm{ClC}_{6} \mathrm{H}_{4}$ 7i: $\mathrm{Ar}^{1}=\mathrm{Ar}^{2}=\mathrm{Ph}$

\section{Scheme 1}

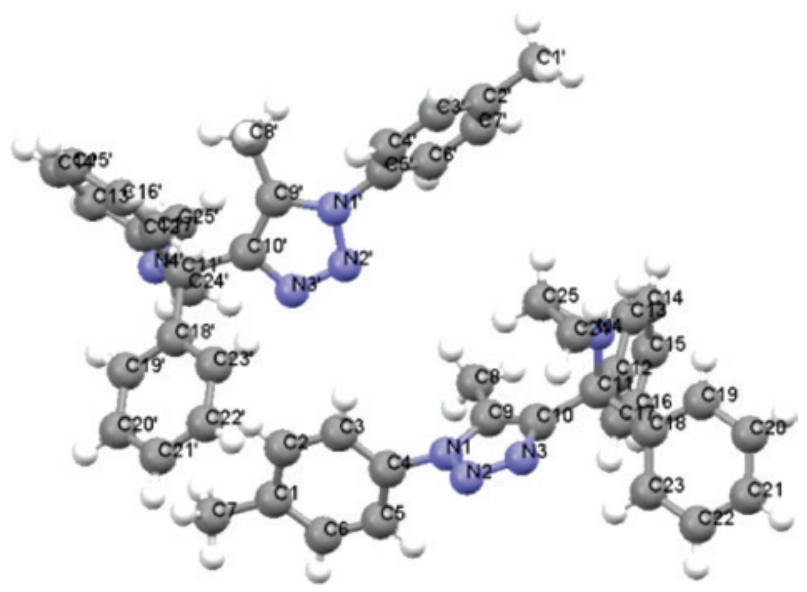

Figure 3 View of the molecular structure of $7 f(C C D C, 2005)$.

4-[(Aziridin-1-yl)diphenylmethyl]-5-methyl-1-(4-chlorophenyl)1H-1,2,3-triazole (7c) White needles; yield $90 \%$; mp $92-94^{\circ} \mathrm{C} ;{ }^{1} \mathrm{H}$ NMR: $\delta 7.50(\mathrm{~m}, 6 \mathrm{H}), 7.50(\mathrm{~m}, 2 \mathrm{H}), 7.26(\mathrm{~m}, 6 \mathrm{H}), 2.08(\mathrm{~s}, 3 \mathrm{H}), 1.80$ (s, 2H), 1.19 (d, $2 \mathrm{H}, J=2 \mathrm{~Hz}) ;{ }^{13} \mathrm{C}$ NMR $\left(\mathrm{CDCl}_{3}\right.$-d1), $\delta$ 146.6, 143.3, 135.3, 134.9, 132.8, 129.5, 129.2, 127.4, 126.8, 126.7, 69.9, 21.7, 10.5; MS: $\mathrm{m} / z 400$ $\left(\mathrm{M}^{+}, 1.2\right), 358$ (11), 178 (5), 154 (32) 152 (100), 11 (26), 77 (8), 75 (11), 51 (5), 43 (7); IR: 3459, 3088, 3059, 2987, 2928, 1738, 1594, 1495, 1445, 1422, 1402, 1371, 1262, 1235, 1202, 1177, 1120, 1090, 1042, 1004, 938, 902, 848, $821,765,744,700,639 \mathrm{~cm}^{-1}$. HRMS (ESI). Calcd for $\mathrm{C}_{24} \mathrm{H}_{21} \mathrm{ClN}_{4},[\mathrm{M}+\mathrm{H}]^{+}$: $m / z$ 401.1528. Found: $m / z$ 401.1532.

4-[(Aziridin-1-yl)-bis(4-chlorophenyl)methyl]-5-methyl-1-ptolyl-1H-1,2,3-triazole (7d) White flakes; yield $89 \%$; mp $86-88^{\circ} \mathrm{C}$; ${ }^{1} \mathrm{H}$ NMR: $\delta 7.48$ (m, 4H), 7.28 (m, 8H), 2.43 (s, 3H), 2.00 (s, 3H), 1.80 (d, $2 \mathrm{H}, J=2 \mathrm{~Hz}), 1.15$ (d, $2 \mathrm{H}, J=2 \mathrm{~Hz}) ;{ }^{13} \mathrm{C}$ NMR ( $\left.\mathrm{CDCl}_{3}-\mathrm{d} 1\right), \delta 144.8,142.1$, 139.6, 133.7, 133.1, 132.8, 130.3, 129.9, 127.8, 125.4, 69.2, 21.7, 21.1, 10.5; MS: $m / z 448\left(\mathrm{M}^{+}, 0.52\right), 406$ (8), 152 (22), 133 (11), 132 (100), 125 (7), 111 (12), 97 (8), 91 (11), 83 (12), 74 (11), 55 (15); IR: 3429, 3060, 2987, 2923, 2856, 1517, 1486, 1443, 1421, 1400, 1264, 1205, 1180, 1094, 1011, 923, 867, 819, $559 \mathrm{~cm}^{-1}$. HRMS (ESI). Calcd for $\mathrm{C}_{25} \mathrm{H}_{22} \mathrm{Cl}_{2} \mathrm{~N}_{4},[\mathrm{M}+\mathrm{H}]^{+}: \mathrm{m} / z$ 449.1294. Found: $m / z$ 449.1299.
4-[(Aziridin-1-yl)-bis(3-chlorophenyl)methyl]-5-methyl-1-p-tolyl$\mathbf{1 H}-1,2,3-$ triazole (7e) White needles; yield $82 \%$; mp $103-105^{\circ} \mathrm{C}$; ${ }^{1} \mathrm{H}$ NMR: $\delta 7.65$ (s, 2H), 7.42 (m, 2H), 7.30 (s, 4H), 7.22 (m, 4H), 2.42 (s, 3H), 1.95 (s, 3H), 1.85 (d, $2 \mathrm{H}, J=2 \mathrm{~Hz}), 1.18$ (d, $2 \mathrm{H}, J=2 \mathrm{~Hz}) ;{ }^{13} \mathrm{C}$ NMR $\left(\mathrm{CDCl}_{3}-\right.$ d1), $\delta 145.8,143.8,139.7,133.8,133.7,133.3,129.9,128.9,128.7,127.3,127.2$, 125.4, 69.4, 21.8, 21.2, 10.6; MS: $m / z 448\left(\mathrm{M}^{+}, 0.54\right), 406$ (5), 133 (10), 132 (100), 91 (30), 65 (13); IR: 3449, 3062, 2990, 2923, 2865, 1735, 1590, 1571, 1515, 1469, 1420, 1368, 1256, 1235, 1203, 1179, 1147, 1121, 1102, 1078, 1048, 1010, 939, 883, 826, 785, 754, 729, 698, $682 \mathrm{~cm}^{-1}$; HRMS (ESI). Calcd for $\mathrm{C}_{25} \mathrm{H}_{22} \mathrm{Cl}_{2} \mathrm{~N}_{4},[\mathrm{M}+\mathrm{H}]^{+}: m / z$ 449.1294. Found: $m / z$ 449.1301.

4-[(Aziridin-1-yl)-diphenylmethyl]-5-methyl-1-p-tolyl-1H-1,2,3triazole (7f) White needles; yield $91 \%$; mp $151-152^{\circ} \mathrm{C}$; ${ }^{1} \mathrm{H}$ NMR: $\delta 7.57$ (m, 4H), 7.37 (m, 10H), 2.42 (s, 3H), 1.98(s, 3H), 1.82 (d, 2H, J=2 Hz), $1.22(\mathrm{~d}, 2 \mathrm{H}, J=2 \mathrm{~Hz}) ;{ }^{13} \mathrm{C}$ NMR $\left(\mathrm{CDCl}_{3}-\mathrm{d} 1\right), \delta 145.7,143.8,139.4,133.9$, 132.9, 129.8, 129.1, 127.4, 126.8, 125.5, 69.9, 21.8, 21.2, 10.5; MS: $\mathrm{m} / \mathrm{z} 380$ $\left(\mathrm{M}^{+}, 1.0\right), 33$ (13), 178 (6), 133 (10), 132 (100), 91 (34), 77 (6), 65 (14); IR: 3430, 3055, 3021, 2983, 2926, 2864, 1594, 1516, 1488, 1444, 1420, 1387, 1259, 1208, 1181, 1120, 1104, 1031, 1006, 987, 935, 898, 859, 838, 818, 798, 767, 748, 700, 662, 640, 553, $529 \mathrm{~cm}^{-1}$. HRMS (ESI). Calcd for $\mathrm{C}_{24} \mathrm{H}_{24} \mathrm{~N}_{4},[\mathrm{M}+\mathrm{H}]^{+}: m / z$ 381.2074. Found: $m / z$ 381.2079.

4-[(Aziridin-1-yl)-bis(4-chlorophenyl)methyl]-5-methyl-1-phenyl-1H-1,2,3-triazole (7g) White flakes; yield 90\%; mp 83-85 $\mathrm{C}$; ${ }^{1} \mathrm{H}$ NMR: $\delta 7.52$ (m, 5H), 7.47 (m, 2H), 7.42 (m, 2H), 7.29 (m, 2H), 7.26 (s, $2 \mathrm{H}), 2.05$ (s, 3H), 1.82 (d, 2H, $J=3 \mathrm{~Hz}), 1.16$ (d, $2 \mathrm{H}, J=3 \mathrm{~Hz}) ;{ }^{13} \mathrm{C}$ NMR (CDCl3-d1), $\delta$ 145.1, 141.9, 136.2, 133.0, 132.9, 130.4, 129.5, 129.4, 127.8, 125.6, 69.3, 21.7, 10.6; MS: $m / z 434\left(\mathrm{M}^{+}, 0.7\right), 394$ (5), 392 (9), 332 (11), 129 (11), 119 (11), 118 (100), 104 (10), 91 (13), 77 (21), 57 (11); IR: 3429, 3060, 2987, 2955, 2924, 2854, 1595, 1485, 1416, 1398, 1264, 1113, 1092, 1072, 1011, 921, 867, 817, 763, 694, 565, $531 \mathrm{~cm}^{-1}$. HRMS (ESI). Calcd for $\mathrm{C}_{24} \mathrm{H}_{20} \mathrm{Cl}_{2} \mathrm{~N}_{4},[\mathrm{M}+\mathrm{H}]^{+}: m / z$ 435.1138. Found: $m / z 435.1144$.

4-[(Aziridin-1-yl)-bis(3-chlorophenyl)methyl]-5-methyl-1-phenyl-1H-1,2,3-triazole (7h) White flakes; yield 40\%; mp 141-143 ${ }^{\circ} \mathrm{C}$; ${ }^{1} \mathrm{H}$ NMR: $\delta 7.65$ (s, 2H), 7.52 (m, 3H), 7.40 (m, 4H), 7.23 (m, 4H), 1.99 (s, 3H), 1.85 (t, 2H, $J=2 \mathrm{~Hz}), 1.19$ (t, $2 \mathrm{H}, J=2 \mathrm{~Hz}) ;{ }^{13} \mathrm{C} \mathrm{NMR}\left(\mathrm{CDCl}_{3}\right.$-d1), $\delta$ 145.7, 144.1, 136.2, 133.8, 133.3, 129.5, 129.4, 128.9, 128.7, 127.3, 127.2, 125.6, 69.4, 21.8, 10.5; MS: $m / z 434\left(\mathrm{M}^{+}, 0.3\right), 176$ (5), 118 (100), 77 (69), 51 (16); IR: 3429, 3058, 2985, 2926, 2844, 1592, 1571, 1501, 1471, 1265, 1119, 1144, 1120, 1100, 1015, 976, 940, 893, 813, 784, 761, 747, 713, $695 \mathrm{~cm}^{-1}$. HRMS (ESI). Calcd for $\mathrm{C}_{24} \mathrm{H}_{20} \mathrm{Cl}_{2} \mathrm{~N}_{4},[\mathrm{M}+\mathrm{H}]^{+}: \mathrm{m} / z 435.1138$. Found: $m / z$ 435.1145.

4-[(Aziridin-1-yl)-diphenylmethyl]-5-methyl-1-phenyl-1H-1,2,3triazole (7i) White needles; yield $89 \%$; mp $203-206^{\circ} \mathrm{C}$; ${ }^{1} \mathrm{H}$ NMR: $\delta$ 7.56 (m, 4H), 7.51 (m, 3H), 7.43 (m, 2H), 7.26 (m, 6H), 2.03 (s, 3H), 1.81 (s, $2 \mathrm{H}), 1.21$ (s, 2H); ${ }^{13} \mathrm{C}$ NMR ( $\mathrm{CDCl}_{3}$-d1), $\delta 145.9,143.6,136.4,132.9,129.3$, 129.1, 127.4, 126.8, 125.6, 69.9, 21.7, 10.5; MS: $\mathrm{m} / z 366\left(\mathrm{M}^{+}, 0.4\right), 324$ (8), 178 (5), 119 (8), 118 (100), 77 (46); IR: 3424, 3070, 3051, 3022, 2988, 2925, 2890, 1594, 1496, 1444, 1416, 1387, 1261, 1207, 1077, 1033, 993, 937, 903, 854, 767, 747, 697, 646, $561 \mathrm{~cm}^{-1}$. HRMS (ESI). Calcd for $\mathrm{C}_{24} \mathrm{H}_{22} \mathrm{~N}_{4}$, $[\mathrm{M}+\mathrm{H}]^{+}: m / z$ 367.1917. Found: $m / z$ 367.1924.

\section{Biological evaluation}

The effects of the synthesized compounds on cell viability were investigated under previously reported conditions with human leukemia cells and human hepatoma G2 cells [20, 21] using the MTT assay. 
Acknowledgments: The authors wish to acknowledge the funding support by the Education Department of Gansu Province (grant 2016B-124) and by the Lanzhou University of Arts and Science (grant 2015GSP05).

\section{References}

[1] Dong, H. R.; Chen, Z. B.; Li, R. S.; Dong, H. S.; Xie, Z. X. Convenient and efficient synthesis of disubstituted piperazine derivatives by catalyst-free, atom-economical and tricomponent domino reactions. RSC Adv. 2015, 5, 10768-10772.

[2] Nguyen, M. H.; Imanishi, M.; Kurogi, T.; Smith, A. B. Total synthesis of (-)-mandelalide a exploiting anion relay chemistry (ARC): identification of a type II ARC/CUCN cross-coupling protocol. J. Am. Chem. Soc. 2016, 138, 3675-3678.

[3] Jamookeeah, C. E.; Beadle, C. D.; Harrity, J. P. A. An enantiospecific approach to triazolylalanine derivatives. Synthesis 2009, 2009, 133-137.

[4] Watson, I. D. G.; Yudin, A. K. Ring-opening reactions of nonactivated aziridines catalyzed by tris(pentafluorophenyl) borane. J. Org. Chem. 2003, 68, 5160-5167.

[5] Nakao, Y.; Fusetani, N. Enzyme inhibitors from marine invertebrates. J. Nat. Prod. 2007, 70, 689-710.

[6] Plaza, A.; Gustchina, E.; Baker, H. L.; Kelly, M.; Bewley, C. A. Mirabamides A-D, depsipeptides from the sponge Siliquariaspongia mirabilis that inhibit HIV-1 fusion. J. Nat. Prod. 2007, 70, 1753-1760.

[7] Sharma, V.; Kelly, G. T.; Foulke-Abel, J.; Watanabe, C. M. H. Aminoacetone as the penultimate precursor to the antitumor agent azinomycin A. Org. Lett. 2009, 11, 4006-4009.

[8] Han, H.; Park, S. B.; Kim, S. K.; Chang, S. Copper - nitrenoid formation and transfer in catalytic olefin aziridination utilizing chelating 2-pyridylsulfonyl moieties. J. Org. Chem. 2008, 73, 2862-2870.

[9] Judd, T. C.; Williams, R. M. A Concise total synthesis of (+)-FR900482 and (+)-FR66979. J. Org. Chem. 2004, 69, 2825-2830.

[10] Galm, U.; Hager, M. H.; Van Lanen, S. G.; Ju, J.; Thorson, J. S.; Shen, B. Antitumor antibiotics: bleomycin, enediynes and mitomycin. Chem. Rev. 2005, 105, 739-758.

[11] Zheng, Y. C.; Duan, Y. C.; Ma, J. L. Triazole-dithiocarbamate based selective lysine specific demethylase 1 (LSD1) inactiva- tors inhibit gastric cancer cell growth, invasion, and migration. J. Med. Chem. 2014, 56, 8543-8560.

[12] Zhang, W. J.; Li, Z.; Zhou, M.; Wu, F.; Hou, X. Y.; Luo, H.; Liu, H.; Han, X.; Yan, G. Y.; Ding, Z. Y.; et al. Synthesis and biological evaluation of 4-(1,2,3-triazol-1-yl)coumarin derivatives as potential antitumor agents. Bioorg. Med. Chem. Lett. 2014, 24, 799-807.

[13] Sambasiva Rao, P.; Kurumurthy, C.; Veeraswamy, B.; Santhosh, K. G.; Poornachandra, Y.; Ganesh, K. C.; Vasamsetti, S. B.; Kotamraju, S.; Narsaiah, B. Synthesis of novel 1,2,3-triazole substituted- $N$-alkyl/aryl nitrone derivatives, their anti-inflammatory and anticancer activity. Eur. J. Med. Chem. 2014, 80, 184-191.

[14] Reck, F.; Zhou, F.; Girardot, M.; Kern, G.; Eyermann, C. J.; Hales, N. J.; Ramsay, R. R.; Gravestock, M. B. Identification of 4-substituted 1,2,3-triazoles as novel oxazolidinone antibacterial agents with reduced activity against monoamine oxidase A. J. Med. Chem. 2005, 48, 499-506.

[15] Liang, C. H.; Yao, S.; Chiu, Y. H.; Leung, P. Y.; Robert, N.; Seddon, J.; Sears, P.; Hwang, C. K.; Ichikawa, Y.; Romero, T. Synthesis and biological activity of new 5-O-sugar modified ketolide and 2-fluoro-ketolide antibiotics. Bioorg. Med. Chem. Lett. 2005, 15, 1307-1310.

[16] Chen, H.; Taylor, J. L.; Abrams, S. R. Design and synthesis of $\beta$-methoxyacrylate analogues via click chemistry and biological evaluations. Bioorg. Med. Chem. Lett. 2007, 17, 1979-1983.

[17] Maurya, S. K.; Gollapalli, D. R.; Kirubakaran, S.; Zhang, M. J.; Johnson, C. R.; Benjamin, N. N.; Hedstrom, L.; Cuny, G. D. Triazole inhibitors of cryptosporidium parvum inosine 5'-monophosphate dehydrogenase. J. Med. Chem. 2009, 52, 4623-4630.

[18] Youcef, R. T.; Santos, M. D.; Roussel, S.; Baltaze, J.; Lubin-Germain, N.; Uziel, J. Huisgen cycloaddition reaction of $\mathrm{C}$-alkynyl ribosides under micellar catalysis: synthesis of ribavirin analogues. J. Org. Chem. 2009, 74, 4318-4323.

[19] Dong, H. S.; Huo, G. Y.; Ma, Z. T. The synthesis of some new (1-aryl-5-methyl-1H-1,2,3-triazol-4-yl) diarylmethanol. Indian J. Chem. Sect B 2008, 47, 171-174.

[20] Wang, H.; Gao, H. H.; Wang, Q.; Gao, Q. X.; Lin, C. J. Special anticancer activity of a new bisalolane sesquiterpene against human leukemia cells in vitro via inducing differentiate. Pharmazie 2007, 62, 699-704.

[21] lyengar, B. S.; Dorr, R. T.; Remers, W. A. Chemical basis for the biological activity of imexon and related cyanoaziridines. J. Med. Chem. 2004, 47, 218-223. 